\title{
Oscillation and variation for the Gaussian Riesz transforms and Poisson integral.*
}

\author{
E. Harboure, R. A. Macías, M. T. Menárguez and J. L. Torrea
}

\begin{abstract}
For the family of truncations of the Gaussian Riesz transforms and Poisson integral we study their rate of convergence through the oscillation and variation operators. More precisely, we search for their $L^{p}(d \gamma)$-boundedness properties, being $d \gamma$ the Gauss measure. We achieve our results by looking at the oscillation and variation operators from a vector valued point of view.
\end{abstract}

\section{Introduction}

Given a family of operators $\mathcal{T}=\left\{T_{\varepsilon}\right\}_{\varepsilon>0}$ for which it is known that there exists $\lim _{\varepsilon \rightarrow 0} T_{\varepsilon} f(x)$ almost everywhere for a certain class of functions $f$, it is classical to measure the speed of convergence of the family by means of quadratic expresions involving differences of the type $\left|T_{\varepsilon} f(x)-T_{\varepsilon^{\prime}} f(x)\right|$, see $[1]$.

Along this line, in [3] the authors study the oscillation operator

$$
\mathcal{O}(\mathcal{T} f)(x)=\left(\sum_{i=1}^{\infty} \sup _{t_{i+1} \leq \varepsilon_{i+1}<\varepsilon_{i} \leq t_{i}}\left|T_{\varepsilon_{i}} f(x)-T_{\varepsilon_{i+1}} f(x)\right|^{2}\right)^{1 / 2}
$$

*Partially supported by Dirección General de Investigación, Ministerio de Ciencia y Tecnología, BFM200204013-C02-02 and European Comission contract HPRN-CT-2001-00273.

First and second authors were partially supported by grants from CONICET and Universidad Nacional del Litoral, Argentina.

First author was partially supported by sabbatical grant SAB2001-0053 from Ministerio de Educación Cultura y Deporte (Spain).

2000 Mathematical Subject Classification:42B15, 42B20, 42B25, 42 C10.

Keywords: Ornstein-Uhlenbeck semigroup, singular integrals, vector valued operators. 
where $\left(t_{i}\right)$ is a fixed sequence which decreases to zero and the family $\mathcal{T}=\left\{T_{\varepsilon}\right\}$ is composed by truncations of the Hilbert transform $\mathcal{H}=\left\{H_{\varepsilon}\right\}$ given by

$$
H_{\varepsilon} f(x)=\int_{|x-y|>\varepsilon} \frac{f(y)}{x-y} d y
$$

They proved that the oscillation operator $\mathcal{O}(\mathcal{H} f)(x)$ satisfies $\|\mathcal{O}(\mathcal{H} f)\|_{L^{p}(\mathbb{R})} \leq c_{p}\|f\|_{L^{p}(\mathbb{R})}$ for $1<p<\infty$ and $|\{x:|\mathcal{O}(\mathcal{H} f)(x)|>\lambda\}| \leq(c / \lambda)\|f\|_{L^{1}(\mathbb{R})}$, see [3, Theorem 1.1].

As an important tool in the proof of the above result they get the same result for the oscillation of the family $\Pi=\left\{p_{\varepsilon}\right\}, p_{\varepsilon}$ being the Poisson integral on $\mathbb{R}^{d}$, see [3, Corollary 2.6]. In the same paper they also consider the $\rho$-variation operator

$$
\mathcal{V}_{\rho}(\mathcal{T} f)(x)=\sup _{\left(\varepsilon_{i}\right) \rightarrow 0}\left(\sum_{i=1}^{\infty}\left|T_{\varepsilon_{i}} f(x)-T_{\varepsilon_{i+1}} f(x)\right|^{\rho}\right)^{1 / \rho},
$$

where $\rho>2$ and the sup is taken over all sequences $\left(\varepsilon_{i}\right)$ decreasing to zero.

As in the case of the oscillation, they obtain strong $(p, p)$ boundedness in the range $1<p<\infty$ and weak type $(1,1)$ boundedness of the $\rho$-variation for the families associated to both operators, the Hilbert transform and the Poisson integral, see [3, Theorem 1.2, Corollary 2.6].

Recently, in the paper [4], the same authors have proved the $(p, p)$ boundedness (with constant depending on the dimension), for $p$ in the range $1<p<\infty$, and also the weak type $(1,1)$ boundedness for the oscillation and the $\rho$-variation $(\rho>2)$ of the truncated Riesz transforms $\left\{K_{\alpha, \varepsilon}\right\}$ in $\mathbb{R}^{d}$, where

$$
K_{\alpha, \varepsilon} f(x)=c_{d} \int_{\left\{y \in \mathbb{R}^{d}:|x-y|>\varepsilon\right\}} \frac{x_{\alpha}-y_{\alpha}}{|x-y|^{d+1}} f(y) d y, \quad x \in \mathbb{R}^{d}, \quad \alpha=1, \ldots, d .
$$

Other previous related results can be found in [11], where a probability approach is used with ideas that go back to Bourgain ([2]).

Finally, by using transference arguments, some weighted strong $(p, p)$ boundedness, for $p$ in the range $1<p<\infty$, have been proved in [7] for the oscillation and the $\rho$-variation $(\rho>2)$ of the truncated Riesz transforms. Moreover in this case the constants do not depend on the dimension $d$.

The purpose of this paper is to prove similar results for the oscillation and $\rho$-variation for operators related to the Ornstein-Ulhenbeck semigroup. In order to state the theorems that we shall prove we need to fix some notation.

Let $\left\{S_{t}\right\}$ be a symmetric diffusion semigroup of operators acting on measurable functions on $\mathbb{R}^{d}$, with a second order differential operator $-L$ as its infinitesimal generator. In this context, as an important tool for studying the solutions of the heat and Laplace equations, the following operators are considered, see [21], 
(1) Maximal operator of the subordinated Poisson semigroup: $P^{*} f(x)=\sup _{t>0}\left|P_{t} f(x)\right|$, where $P_{t}$ is defined by the following subordination formula

$$
P_{t} f(x)=\frac{1}{\sqrt{2 \pi}} \int_{0}^{\infty} t e^{-t^{2} / 4 s} S_{s} f(x) s^{-3 / 2} d s
$$

(2) Riesz transforms: For $1 \leq \alpha \leq d$,

$$
R_{\alpha} f(x)=\frac{\partial}{\partial x_{\alpha}} L^{-1 / 2} f(x) .
$$

An expression for $L^{-1 / 2}$ can be derived from the identity $s^{-\beta}=\frac{1}{\Gamma(\beta)} \int_{0}^{\infty} t^{\beta-1} e^{-t s} d t$.

In the classical Euclidean case where $L=-\Delta$ and $S_{t}$ is the Gauss-Weierstrass semigroup, all of these operators are well known. We refer to [22] for their $L^{p}(d x)$-boundedness properties, where $d x$ is the Lebesgue measure in $\mathbb{R}^{d}$. However, since the technique we shall use involves comparison with the Ornstein-Uhlenbeck semigroup given below, we will rather deal with the semigroup $W_{t}$ whose infinitesimal generator is $-\frac{1}{2} \Delta$, that is

$$
W_{t} f(x)=(2 \pi t)^{-d / 2} \int_{\mathbb{R}^{d}} e^{-\frac{|x-y|^{2}}{2 t}} f(y) d y .
$$

Anyway we notice that the operators (1) and (2) defined above are modified only in a constant after this change in the infinitesimal generator.

In this case the Riesz transforms can be viewed as principal values of the integrals against the kernels $K_{\alpha}(x-y)=c_{d}\left(x_{\alpha}-y_{\alpha}\right)|x-y|^{-d-1}$, which appear as the corresponding partial derivatives of the kernel associated to the $\left(-\frac{1}{2} \Delta\right)^{-1 / 2}$ operator. Moreover the maximal operators

$$
K_{\alpha}^{*} f(x)=\sup _{\varepsilon>0}\left|K_{\alpha, \varepsilon} f(x)\right|=\sup _{\varepsilon>0}\left|\int_{\mathbb{R}^{d}} K_{\alpha, \varepsilon}(x-y) f(y) d y\right|, \quad 1 \leq \alpha \leq d,
$$

where $K_{\alpha, \varepsilon}(x-y)=K_{\alpha}(x-y) \chi_{\{|x-y|>\varepsilon\}}(x, y)$, are bounded on $L^{p}(d x), 1<p<\infty$, and of weak type $(1,1)$. This implies both the almost everywhere convergence of $K_{\alpha, \varepsilon} f$ for $f \in L^{p}(d x), 1 \leq p<\infty$ and the convergence in $L^{p}(d x), 1<p<\infty$, or the weak- $L^{1}(d x)$ convergence, see [22].

In the case where $L=-\frac{1}{2} \Delta+x \cdot \nabla, S_{t}$ is the Ornstein-Uhlenbeck semigroup $O_{t}$ given by

$$
O_{t} f(x)=\left(\pi\left(1-e^{-2 t}\right)\right)^{-d / 2} \int_{\mathbb{R}^{d}} e^{-\frac{\left|e^{-t} x-y\right|^{2}}{1-e^{-2 t}}} f(y) d y
$$

(see for example [20]). In this setting the natural measure is the Gauss measure $d \gamma(x)=$ $e^{-|x|^{2}} d x$. The above operators have been intensively studied in the last twenty years by several 
authors. For the $L^{p}(d \gamma)$-boundedness of the maximal operator $O^{*}$ we refer to [21] and [20], for the Riesz transforms, $R_{\alpha}$, see [15], [8], [14], [18], [5], [9], [23] and [6].

As in the classical case, the Riesz transforms can be viewed as principal values of integrals, this time against the kernels

$$
R_{\alpha}(x, y)=c_{d} \int_{0}^{\infty} t^{-1 / 2} e^{-t} \frac{e^{-t} x_{\alpha}-y_{\alpha}}{\left(1-e^{-2 t}\right)^{d / 2+1}} \exp \left(-\frac{\left|e^{-t} x-y\right|^{2}}{1-e^{-2 t}}\right) d t
$$

which appear as the corresponding derivatives of the kernel associated to the $\left(-\frac{1}{2} \Delta+x \cdot \nabla\right)^{-1 / 2}$ operator. Namely, for functions $f$ which are good enough, we have

$$
R_{\alpha} f(x)=\lim _{\varepsilon \rightarrow 0} R_{\alpha, \varepsilon} f(x), \quad \text { a.e. } x,
$$

where $R_{\alpha, \varepsilon}(x, y)=R_{\alpha}(x, y) \chi_{\{|x-y|>\varepsilon\}}(x, y)$.

Regarding the speed of convergence of the families $\mathcal{R}_{\alpha}=\left\{R_{\alpha, \varepsilon}\right\}, 1 \leq \alpha \leq d$, we shall prove the following result:

Theorem 1.1 Let $2<\rho<\infty$. Given $p$ in the range $1<p<\infty$, then

$$
\left\|\mathcal{O}\left(\mathcal{R}_{\alpha} f\right)\right\|_{L^{p}\left(\mathbb{R}^{d}, d \gamma\right)} \leq C\|f\|_{L^{p}\left(\mathbb{R}^{d}, d \gamma\right)}, \quad \alpha=1, \ldots, d,
$$

and

$$
\left\|\mathcal{V}_{\rho}\left(\mathcal{R}_{\alpha} f\right)\right\|_{L^{p}\left(\mathbb{R}^{d}, d \gamma\right)} \leq C\|f\|_{L^{p}\left(\mathbb{R}^{d}, d \gamma\right)}, \alpha=1, \ldots, d .
$$

Moreover we have

$$
\gamma\left(\left\{x:\left|\mathcal{O}\left(\mathcal{R}_{\alpha} f\right)(x)\right|>\lambda\right\}\right) \leq \frac{C}{\lambda} \int_{\mathbb{R}^{d}}|f(x)| d \gamma(x), \alpha=1, \ldots, d,
$$

and

$$
\gamma\left(\left\{x:\left|\mathcal{V}_{\rho}\left(\mathcal{R}_{\alpha} f\right)(x)\right|>\lambda\right\}\right) \leq \frac{C}{\lambda} \int_{\mathbb{R}^{d}}|f(x)| d \gamma(x), \alpha=1, \ldots, d
$$

We remark that the $L^{p}(d \gamma)$ boundedness of the operator $O^{*}$ mentioned above, implies the corresponding property for the maximal operator of the Poisson integral. In turn this implies the almost everywhere convergence of the family $\mathcal{P}=\left\{P_{t}\right\}$ when acting over functions in $L^{p}(d \gamma), 1 \leq p<\infty$.

Concerning the speed of convergence of the family $\mathcal{P}$ we have the following result:

Theorem 1.2 Let $2<\rho<\infty$. Given $p$ in the range $1<p<\infty$, then

$$
\|\mathcal{O}(\mathcal{P} f)\|_{L^{p}\left(\mathbb{R}^{d}, d \gamma\right)} \leq C\|f\|_{L^{p}\left(\mathbb{R}^{d}, d \gamma\right)},
$$

and

$$
\left\|\mathcal{V}_{\rho}(\mathcal{P} f)\right\|_{L^{p}\left(\mathbb{R}^{d}, d \gamma\right)} \leq C\|f\|_{L^{p}\left(\mathbb{R}^{d}, d \gamma\right)}
$$


Moreover

$$
\gamma(\{x:|\mathcal{O}(\mathcal{P} f)(x)|>\lambda\}) \leq \frac{C}{\lambda} \int_{\mathbb{R}^{d}}|f(x)| d \gamma(x),
$$

and

$$
\gamma\left(\left\{x:\left|\mathcal{V}_{\rho}(\mathcal{P} f)(x)\right|>\lambda\right\}\right) \leq \frac{C}{\lambda} \int_{\mathbb{R}^{d}}|f(x)| d \gamma(x)
$$

The organization of this paper is the following. In section 2 we present some known facts from the Gaussian analysis that we need for our purposes. Also, following [7], we present the oscillation and $\rho$-variation of a family of operators, see (2.8) and (2.10), from a vector valued point of view. This setting allows us to apply the general machinery for Gaussian operators just described. In section 3 we prove the results concerning the Riesz transforms, while section 4 is devoted to the Poisson integral.

\section{Preliminaries}

In proving the Theorems, following the technique initiated in [15], we will use systematically a partition of the operators into their local and global parts, according to some particular region, where Lebesgue and Gauss measure are equivalent, and its complement. In the literature, depending on the operator under consideration, two kinds of regions have been used, either

$$
N_{t}=\left\{(x, y):|x-y|<\frac{t}{1+|x|+|y|}\right\}, \text { for some fixed } t>0
$$

or

$$
\mathcal{N}_{t}=\left\{(x, y):|x-y|<\min \left(t, \frac{t}{|x|}\right)\right\}, \text { for some fixed } t>0
$$

See [20], [5], [17] and [6].

For the global part of the operators we want to make use of estimates given by different authors for the kernels in the complement of regions like $\mathcal{N}_{t}$, while for the local part sometimes it will be convenient to work with the $N_{t}$-regions. However, from the relationship:

$$
N_{t} \subset \mathcal{N}_{t} \subset N_{t(t+3)}
$$

it follows that we may use one region or the other when estimating positive functions. On the sequel we shall denote by $N$ the region $N_{t}$ for $t=d(d+3)$ and by $\tilde{N}$ that corresponding to $N_{2 d(d+3)}$, when working on $\mathbb{R}^{d}$.

Now we remark that the operators we will handle, the oscillation and variation, are not linear; however, as we shall see, they can be viewed as linear operators taking values on 
appropriate Banach spaces. For this reason we shall consider a class of operators like those given in [6], but this time in a vector valued setting.

Given $B_{1}, B_{2}$ Banach spaces, let $d \mu$ denote either the Lebesgue or the Gauss measure on $\mathbb{R}^{d}$. Let $T$ be a linear operator defined in $L_{0, B_{1}}^{\infty}$, the space of $B_{1}$-valued, compactly supported and essentially bounded functions on $\mathbb{R}^{d}$, into the space of $B_{2}$-valued and strongly measurable functions on $\mathbb{R}^{d}$, satisfying :

(a) $T$ extends to a bounded operator either from $L_{B_{1}}^{q}(d \mu)$ into $L_{B_{2}}^{q}(d \mu)$ for some $q, 1<$ $q<\infty$, or from $L_{B_{1}}^{1}(d \mu)$ into weak- $L_{B_{2}}^{1}(d \mu)$.

(b) There exists a $\mathcal{L}\left(B_{1}, B_{2}\right)$-valued measurable function $K$, defined on the complement of the diagonal in $\mathbb{R}^{d} \times \mathbb{R}^{d}$, such that for every function $f$ in $L_{0, B_{1}}^{\infty}$

$$
T f(x)=\int K(x, y) f(y) d y,
$$

for all $x$ outside the support of $f$.

(c) The function $K$ satisfies the estimate

$$
|K(x, y)| \leq \frac{C}{|x-y|^{d}}
$$

for all $(x, y)$ in the local region $N, x \neq y$.

As in [6] we introduce the following definitions.

For an operator $T$ as above, given $\varphi$ a smooth function on $\mathbb{R}^{d} \times \mathbb{R}^{d}$ such that $\varphi(x, y)=1$ if $(x, y) \in N, \varphi(x, y)=0$ for $(x, y) \notin \tilde{N}=N_{2 d(d+3)}$ and

$$
\left|\partial_{x} \varphi(x, y)\right|+\left|\partial_{y} \varphi(x, y)\right| \leq C|x-y|^{-1} \text { if } x \neq y .
$$

We define the global and the local parts of the operator $T$ by

$$
\begin{aligned}
T_{\text {glob }} f(x) & =\int K(x, y)(1-\varphi(x, y)) f(y) d y, \\
T_{l o c} f(x) & =T f(x)-T_{\text {glob }} f(x),
\end{aligned}
$$

and accordingly we shall call $K_{g l o b}$ and $K_{l o c}$ their kernels.

We shall use the following results, see Proposition 3.4 and Lemma 3.6 in [6]. Even though the authors give the proof in the scalar case, the same arguments work in the vector valued setting.

Proposition 2.1 If $T$ is an operator satisfying conditions (a),(b) and (c), then $T_{\text {loc }}$ inherits from $T$ either the $L^{q}$ - boundedness or the weak type $(1,1)$ as the case might be. Besides the corresponding boundedness holds for both Lebesgue and Gauss measure. 
We shall say that an operator $T$ defined on $L_{0, B_{1}}^{\infty}$ into the space of $B_{2}$-valued strongly measurable functions is local if its associated kernel in the sense of (b) is supported in $\tilde{N}$.

Proposition 2.2 If $S$ is a local operator, then strong type $(p, p), 1 \leq p<\infty$ for Lebesgue and Gauss measures are equivalent. The same holds for weak type $(p, p)$.

In order to apply the above results and following [7] we shall give vector valued versions of the Oscillation and Variation of a family of operators. To simplify the description of the Oscillation we introduce the equivalent operator

$$
\mathcal{O}^{\prime}(\mathcal{T} f)(x)=\left(\sum_{i=1}^{\infty} \sup _{t_{i+1}<\delta_{i} \leq t_{i}}\left|T_{t_{i+1}} f(x)-T_{\delta_{i}} f(x)\right|^{2}\right)^{1 / 2},
$$

that clearly satisfies

$$
\mathcal{O}^{\prime}(\mathcal{T} f)(x) \sim \mathcal{O}(\mathcal{T} f)(x), \quad \text { a.e.x. }
$$

Consequently it will be enough to prove Theorem 1.1 and Theorem 1.2 for the operator $\mathcal{O}^{\prime}$ instead of $\mathcal{O}$.

Let us denote by $E$ the mixed normed Banach space of two variable functions $h$ defined on $\mathbb{R} \times \mathbb{N}$ such that

$$
\|h\|_{E} \equiv\left(\sum_{i}\left(\sup _{s}|h(s, i)|\right)^{2}\right)^{1 / 2}<\infty .
$$

Let $\mathcal{T}=\left\{T_{t}\right\}_{t>0}$ be a family of operators defined on $L^{p}\left(\mathbb{R}^{d}, d \mu\right)$, for some $p$ in the range $1 \leq p<\infty$. Given a fixed decreasing sequence $t_{i} \searrow 0$, let $J_{i}=\left(t_{i+1}, t_{i}\right]$ and define the operator $U(\mathcal{T}): f \longrightarrow U(\mathcal{T}) f$, where $U(\mathcal{T}) f$ is the $E$-valued function given by

$$
U(\mathcal{T}) f(x)=\left\{T_{t_{i+1}} f(x)-T_{s} f(x)\right\}_{s \in J_{i}, i \in \mathbb{N}} .
$$

Here the expression $\left\{T_{t_{i+1}} f(x)-T_{s} f(x)\right\}_{s \in J_{i}, i \in \mathbb{N}}$ is a convenient abbreviation for the element of $E$ given by

$$
(s, i) \longrightarrow\left(T_{t_{i+1}} f(x)-T_{s} f(x)\right) \chi_{J_{i}}(s)
$$

Then

$$
\mathcal{O}^{\prime}(\mathcal{T} f)(x)=\left\|\left\{T_{t_{i+1}} f(x)-T_{s} f(x)\right\}_{s \in J_{i}, i \in \mathbb{N}}\right\|_{E}=\|U(\mathcal{T}) f(x)\|_{E}
$$

Let $\Theta=\left\{\varepsilon: \varepsilon=\left\{\varepsilon_{i}\right\}, \varepsilon_{i} \in \mathbb{R}, \varepsilon_{i} \searrow 0\right\}$. We consider the set $\mathbb{N} \times \Theta$ and denote by $F_{\rho}, 1 \leq \rho<\infty$, the mixed normed space of two variable functions $g(i, \varepsilon)$ such that

$$
\|g\|_{F_{\rho}} \equiv \sup _{\varepsilon}\left(\sum_{i}|g(i, \varepsilon)|^{\rho}\right)^{1 / \rho}<\infty .
$$


For a family $\mathcal{T}$ as above, we also consider the operator $V(\mathcal{T}): f \longrightarrow V(\mathcal{T}) f$, acting on functions $f$ belonging to $L^{p}\left(\mathbb{R}^{d}, d \mu\right)$, and $V(\mathcal{T}) f$ being the $F_{\rho}$-valued function given by

$$
V(\mathcal{T}) f(x)=\left\{T_{\varepsilon_{i+1}} f(x)-T_{\varepsilon_{i}} f(x)\right\}_{\varepsilon \in \Theta} .
$$

Here the expression $\left\{T_{\varepsilon_{i+1}} f(x)-T_{\varepsilon_{i}} f(x)\right\}_{\varepsilon \in \Theta}$ is an abbreviation for the element of $F_{\rho}$ given by

$$
(i, \varepsilon)=\left(i,\left\{\varepsilon_{k}\right\}\right) \longrightarrow T_{\varepsilon_{i+1}} f(x)-T_{\varepsilon_{i}} f(x) .
$$

As in the case of the oscillation operator it is clear that

$$
\mathcal{V}_{\rho}(\mathcal{T} f)=\|V(\mathcal{T}) f\|_{F_{\rho}} .
$$

Proposition 2.3 The identities (2.8) and (2.10) imply that each of the inequalities

$$
\left\|\mathcal{O}^{\prime}(\mathcal{T} f)\right\|_{L^{p}\left(\mathbb{R}^{d}, d \mu\right)} \leq C\|f\|_{L^{p}\left(\mathbb{R}^{d}, d \mu\right)}
$$

and

$$
\mu\left(\left\{x: \mathcal{O}^{\prime}(\mathcal{T} f)(x)>\lambda\right\}\right) \leq \frac{C}{\lambda}\|f\|_{L^{1}\left(\mathbb{R}^{d}, d \mu\right)}
$$

is equivalent to the corresponding inequality

$$
\|U(\mathcal{T}) f\|_{L_{E}^{p}\left(\mathbb{R}^{d}, d \mu\right)} \leq C\|f\|_{L^{p}\left(\mathbb{R}^{d}, d \mu\right)}
$$

and

$$
\mu\left(\left\{x:\|U(\mathcal{T}) f(x)\|_{E}>\lambda\right\}\right) \leq \frac{C}{\lambda}\|f\|_{L^{1}\left(\mathbb{R}^{d}, d \mu\right)} .
$$

$A$ similar result holds for $\mathcal{V}_{\rho}(\mathcal{T} f)$ and $\|V(\mathcal{T}) f\|_{F_{\rho}}$.

Remark 2.1 In the case that the family $\mathcal{T}=\left\{T_{t}\right\}$ is such that each operator $T_{t}$ has an associated kernel $M_{t}$, the operator $U(\mathcal{T})$ has also an associated kernel $\mathcal{U}$, where $\mathcal{U}(x, y)$ is the element of $E$ given by

$$
(s, i) \rightarrow \mathcal{U}(x, y)(s, i)=\left(M_{t_{i+1}}(x, y)-M_{s}(x, y)\right) \chi_{J_{i}}(s),
$$

in other words

$$
U(\mathcal{T}) f(x)=\int \mathcal{U}(x, y) f(y) d y=\int\left\{\left(M_{t_{i+1}}(x, y)-M_{s}(x, y)\right) \chi_{J_{i}}(s)\right\} f(y) d y
$$

Analogous formulas can be given for the variation.

For a family $\mathcal{T}=\left\{T_{t}\right\}$ as in the above remark, we define its global and local parts by

$$
\mathcal{T}_{\text {glob }}=\left\{T_{t, g l o b}\right\}, \quad \mathcal{T}_{l o c}=\left\{T_{t, l o c}\right\}
$$


Lemma 2.1 Given the family $\mathcal{T}=\left\{T_{t}\right\}$, such that each operator $T_{t}$ has an associated kernel $M_{t}$, we have

$$
U(\mathcal{T})_{l o c} f(x)=U\left(\mathcal{T}_{l o c}\right) f(x)
$$

and

$$
U(\mathcal{T})_{g l o b} f(x)=U\left(\mathcal{T}_{\text {glob }}\right) f(x) .
$$

Parallel properties can be shown for the operator $V$ associated to the variation.

Proof By using (2.11) we have

$$
\begin{aligned}
U(\mathcal{T}) f(x) & =\left\{T_{t_{i+1}} f(x)-T_{s} f(x)\right\}_{s \in J_{i}, i \in \mathbb{N}} \\
& =\left\{\int_{\mathbb{R}^{d}}\left(M_{t_{i+1}}(x, y)-M_{s}(x, y)\right) f(y) d y\right\}_{s \in J_{i}, i \in \mathbb{N}} \\
& =\int_{\mathbb{R}^{d}} \mathcal{U}(x, y) f(y) d y .
\end{aligned}
$$

Therefore

$$
\begin{aligned}
U(\mathcal{T})_{\text {glob }} f(x) & =\int_{\mathbb{R}^{d}} \mathcal{U}(x, y)(1-\varphi(x, y)) f(y) d y \\
& =\left\{\int_{\mathbb{R}^{d}}\left(M_{t_{i+1}}(x, y)-M_{s}(x, y)\right)(1-\varphi(x, y)) f(y) d y\right\}_{s \in J_{i}, i \in \mathbb{N}} \\
& =\left\{T_{t_{i+1}, g l o b} f(x)-T_{s, g l o b} f(x)\right\}_{s \in J_{i}, i \in \mathbb{N}} \\
& =U\left(\mathcal{T}_{\text {glob }}\right) f(x) .
\end{aligned}
$$

Hence, as $U$ is linear, the lemma is proved.

\section{$3 \quad$ Riesz Transforms}

In this section we shall deal with the family $\mathcal{R}_{\alpha}=\left\{R_{\alpha, \varepsilon}\right\}_{\varepsilon>0}, 1 \leq \alpha \leq d$, proving the results stated in Theorem 1.1. We start by recalling a known result for the global part of the Riesz transforms.

Lemma 3.1 The global part of the Riesz transforms kernels satisfies

$$
\left|R_{\alpha, \varepsilon, g l o b}(x, y)\right| \leq\left|R_{\alpha, g l o b}(x, y)\right| \leq Q(x, y) \quad \alpha=1, \ldots, d,
$$

for some nonnegative kernel $Q(x, y)$, independent of $\varepsilon$, supported in $N^{c}$ and such that its associated integral operator is of weak type $(1,1)$ and strong type $(p, p), 1<p<\infty$, with respect to the Gauss measure. 
For a proof see [5] and [17].

Remark 3.1 These results imply in particular that the integrals $\int R_{\alpha, \varepsilon, g l o b}(x, y) f(y) d y$ and $\int R_{\alpha, g l o b}(x, y) f(y) d y$ are finite almost everywhere, for every $f \in L^{1}(d \gamma)$.

The next lemma shows that the local part of the oscillation and variation operators for the Gaussian Riesz transforms are, up to a good operator, the same as the corresponding local oscillation and variation of the families $\mathcal{K}_{\alpha}$ associated to the Euclidean Riesz transforms.

Lemma 3.2 For any $\alpha=1, \ldots, d$, we have

$$
\left\|U\left(\mathcal{R}_{\alpha}\right)_{l o c} f(x)-U\left(\mathcal{K}_{\alpha}\right)_{l o c} f(x)\right\|_{E} \leq \int_{\mathbb{R}^{d}} L(x, y)|f(y)| d y
$$

and

$$
\left\|V\left(\mathcal{R}_{\alpha}\right)_{l o c} f(x)-V\left(\mathcal{K}_{\alpha}\right)_{l o c} f(x)\right\|_{F_{\rho}} \leq \int_{\mathbb{R}^{d}} L(x, y)|f(y)| d y,
$$

where $L$ is a nonnegative kernel supported on $\tilde{N}$ and satisfying

$$
\sup _{x} \int_{\mathbb{R}^{d}} L(x, y) d y<\infty \quad \text { and } \quad \sup _{y} \int_{\mathbb{R}^{d}} L(x, y) d x<\infty .
$$

Consequently the integral operator associated to $L$ is of strong type $(p, p), 1 \leq p<\infty$, with respect to either Lebesgue or Gauss measure.

In order to prove the result above we need the following technical lemma, see [10] Lemma 3.4 .

Lemma 3.3 For $(x, y) \in \tilde{N}$ we have the following estimates:

$$
D_{\alpha}(x, y)=\int_{0}^{\infty}\left|\frac{\partial}{\partial x_{\alpha}}\left(O_{t}(x, y)-W_{t}(x, y)\right)\right| \frac{d t}{t^{1 / 2}} \leq C \frac{1+|x|}{|x-y|^{d-1}},
$$

In particular the above kernels when truncated by $\chi_{\tilde{N}}(x, y)$ satisfy conditions like 3.14.

Proof of Lemma 3.2 We shall give the proof only for the operator $U$ associated to the oscillation. The result for the variation follows exactly in the same way.

The kernel for $R_{\alpha, \varepsilon, l o c}-K_{\alpha, \varepsilon, l o c}$ is given by

$$
c \chi_{\{|x-y|>\varepsilon\}}(x, y) \varphi(x, y) \int_{0}^{\infty} \frac{\partial}{\partial x_{\alpha}}\left(O_{t}-W_{t}\right)(x, y) \frac{d t}{t^{1 / 2}} .
$$


By using Lemma 2.1 we have

$$
\begin{aligned}
& \left\|U\left(\mathcal{R}_{\alpha}\right)_{l o c} f(x)-U\left(\mathcal{K}_{\alpha}\right)_{l o c} f(x)\right\|_{E}=\left\|U\left(\mathcal{R}_{\alpha, l o c}\right) f(x)-U\left(\mathcal{K}_{\alpha, l o c}\right) f(x)\right\|_{E} \\
& =\left\|\left\{\int_{\mathbb{R}^{d}}\left(R_{\alpha, l o c}(x, y)-K_{\alpha, l o c}(x, y)\right) \chi_{\left\{t_{i+1}<|x-y| \leq s\right\}}(x, y) f(y) d y\right\}_{s \in J_{i}, i \in \mathbb{N}}\right\|_{E} \\
& \leq c \int_{\mathbb{R}^{d}} D_{\alpha}(x, y) \varphi(x, y)\left\|\left\{\chi_{\left\{t_{i+1}<|x-y| \leq s\right\}}(x, y)\right\}_{s \in J_{i}, i \in \mathbb{N}}\right\|_{E}|f(y)| d y \\
& \leq c \int_{\mathbb{R}^{d}} D_{\alpha}(x, y) \varphi(x, y)|f(y)| d y .
\end{aligned}
$$

Then Lemma 3.2 follows from the estimates given in Lemma 3.3.

Proof of Theorem 1.1 Again we give the proof for the oscillation, since the estimates and results we will use remain valid for the variation with the obvious changes.

By using formula (2.8) it is enough to prove that

$$
\left\|U\left(\mathcal{R}_{\alpha}\right) f\right\|_{L_{E}^{p}\left(\mathbb{R}^{d}, d \gamma\right)} \leq C\|f\|_{L^{p}\left(\mathbb{R}^{d}, d \gamma\right)}, \quad \alpha=1, \ldots, d,
$$

and

$$
\gamma\left(\left\{x:\left\|U\left(\mathcal{R}_{\alpha}\right) f(x)\right\|_{E}>\lambda\right\}\right) \leq \frac{C}{\lambda} \int_{\mathbb{R}^{d}}|f(x)| d \gamma(x), \alpha=1, \ldots, d .
$$

Moreover, as $U\left(\mathcal{R}_{\alpha}\right) f=U\left(\mathcal{R}_{\alpha}\right)_{\text {glob }} f+U\left(\mathcal{R}_{\alpha}\right)_{\text {loc }} f$ it suffices to prove the above inequalities for $U\left(\mathcal{R}_{\alpha}\right)_{\text {glob }} f$ and $U\left(\mathcal{R}_{\alpha}\right)_{l o c} f$.

By using Lemma 2.1, we have

$$
\begin{aligned}
\left\|U\left(\mathcal{R}_{\alpha}\right)_{g l o b} f(x)\right\|_{E} & =\left\|\left\{\int_{\mathbb{R}^{d}} \mathcal{R}_{\alpha, g l o b}(x, y) \chi_{\left\{t_{i+1}<|x-y| \leq s\right\}}(x, y) f(y) d y\right\}_{s \in J_{i}, i \in \mathbb{N}}\right\|_{E} \| \\
& \leq \int_{\mathbb{R}^{d}} Q(x, y) \chi_{N^{c}}(x, y)\left\|\left\{\chi_{\left\{t_{i+1}<|x-y| \leq s\right\}}(x, y)\right\}_{s \in J_{i}, i \in \mathbb{N}}\right\|_{E}|f(y)| d y \\
& \leq \int_{\mathbb{R}^{d}} Q(x, y) \chi_{N^{c}}(x, y)|f(y)| d y,
\end{aligned}
$$

where we have also used Lemma 3.1. Moreover the same Lemma 3.1 guarantees that inequalities (3.15) and (3.16) hold for the global part.

Now, for the local part we have

$$
\left\|U\left(\mathcal{R}_{\alpha}\right)_{l o c} f(x)\right\|_{E} \leq\left\|U\left(\mathcal{R}_{\alpha}\right)_{l o c} f(x)-U\left(\mathcal{K}_{\alpha}\right)_{l o c} f(x)\right\|_{E}+\left\|U\left(\mathcal{K}_{\alpha}\right)_{l o c} f(x)\right\|_{E} .
$$

By Lemma 3.2 we know that the first term satisfies inequalities (3.15) and (3.16). Now we study the second one. It was shown in [4], see also [7], that

$$
\left\|\mathcal{O}\left(\mathcal{K}_{\alpha} f\right)\right\|_{L^{p}\left(\mathbb{R}^{d}, d x\right)} \leq c_{p}\|f\|_{L^{p}\left(\mathbb{R}^{d}, d x\right)}, \alpha=1, \ldots, d,
$$

and

$$
\left|\left\{x:\left|\mathcal{O}\left(\mathcal{K}_{\alpha} f\right)(x)\right|>\lambda\right\}\right| \leq \frac{C}{\lambda} \int_{\mathbb{R}^{d}}|f(x)| d x, \alpha=1, \ldots, d .
$$


That is, by using (2.8),

$$
\left\|U\left(\mathcal{K}_{\alpha}\right) f\right\|_{L_{E}^{p}\left(\mathbb{R}^{d}, d x\right)} \leq c_{p}\|f\|_{L^{p}\left(\mathbb{R}^{d}, d x\right)}, \alpha=1, \ldots, d,
$$

and

$$
\left|\left\{x:\left\|U\left(\mathcal{K}_{\alpha}\right) f(x)\right\|_{E}>\lambda\right\}\right| \leq \frac{C}{\lambda} \int_{\mathbb{R}^{d}}|f(x)| d x, \alpha=1, \ldots, d .
$$

The kernel of the operator $U\left(\mathcal{K}_{\alpha}\right)$ is given by

$$
\mathcal{U}(x, y)=\left\{\left(K_{\alpha, t_{i+1}}(x, y)-K_{\alpha, s}(x, y)\right) \chi_{J_{i}}(s)\right\}=\left\{c_{\alpha} \frac{x_{\alpha}-y_{\alpha}}{|x-y|^{d+1}} \chi_{\left\{t_{i+1}<|x-y| \leq s\right\}}(x, y) \chi_{J_{i}}(s)\right\},
$$

then

$$
\|\mathcal{U}(x, y)\|_{E} \leq \frac{1}{|x-y|}\left\|\left\{\chi_{\left\{t_{i+1}<|x-y| \leq s\right\}}(x, y) \chi_{J_{i}}(s)\right\}\right\|_{E} \leq C_{\alpha} \frac{1}{|x-y|^{d}} .
$$

Therefore the operator $U\left(\mathcal{K}_{\alpha}\right)$ satisfies the hypothesis of Proposition 2.1, which together with Proposition 2.2 give

$$
\left\|U\left(\mathcal{K}_{\alpha}\right)_{l o c} f\right\|_{L_{E}^{p}\left(\mathbb{R}^{d}, d \gamma\right)} \leq C\|f\|_{L^{p}\left(\mathbb{R}^{d}, d \gamma\right)}
$$

and

$$
\gamma\left(\left\{x:\left\|U\left(\mathcal{K}_{\alpha}\right)_{l o c} f(x)\right\|_{E}>\lambda\right\}\right) \leq \frac{C_{\alpha}}{\lambda} \int_{\mathbb{R}^{d}}|f(x)| d \gamma(x) .
$$

Then the result follows for $p=1$. Finally, for $1<p<\infty$, it is known that in any dimension (see [4] and [7])

$$
\left\|U\left(\mathcal{K}_{\alpha}\right) f\right\|_{L_{E}^{p}\left(\mathbb{R}^{d}, d x\right)} \leq c_{p}\|f\|_{L^{p}\left(\mathbb{R}^{d}, d x\right)}, \alpha=1, \ldots, d .
$$

Proceeding as in the one dimensional case we arrive to the desired estimate for $\left\|U\left(\mathcal{K}_{\alpha, l o c}\right) f(x)\right\|_{E}$.

\section{Poisson integral}

In this section we want to study the oscillation and variation of the Poisson semigroup subordinated to the Ornstein-Uhlenbeck semigroup $O_{t}$, as in (1.2), this is

$$
P_{t} f(x)=\frac{1}{\sqrt{2 \pi}} \int_{0}^{\infty} t e^{-t^{2} / 4 s} O_{s} f(x) s^{-3 / 2} d s .
$$

We shall also need some properties of the classical Euclidean Poisson semigroup, that is

$$
p_{t} f(x)=\frac{1}{\sqrt{2 \pi}} \int_{0}^{\infty} t e^{-t^{2} / 4 s} W_{s} f(x) s^{-3 / 2} d s=c_{d} \int_{\mathbb{R}^{d}} \frac{t}{\left(t^{2}+|x-y|^{2}\right)^{\frac{d+1}{2}}} f(y) d y .
$$

For the families $\mathcal{P}=\left\{P_{t}\right\}, \Pi=\left\{p_{t}\right\}$ we consider the corresponding vector valued operators

$$
U(\mathcal{P}), V(\mathcal{P}), U(\Pi) \text { and } V(\Pi),
$$

associated to the oscillation and variation, as given in (2.7) and (2.9).

In the next lemma we compare the local parts of these operators associated to $\mathcal{P}$ and $\Pi$. 


\section{Lemma 4.1}

$$
\left\|U(\mathcal{P})_{l o c} f(x)-U(\Pi)_{l o c} f(x)\right\|_{E} \leq \int_{\mathbb{R}^{d}} L P(x, y) f(y) d y+2\left(P_{l o c}^{*} f(x)+p_{l o c}^{*} f(x)\right)
$$

and

$$
\left\|V(\mathcal{P})_{l o c} f(x)-V(\Pi)_{l o c} f(x)\right\|_{F_{\rho}} \leq \int_{\mathbb{R}^{d}} L P(x, y) f(y) d y+2\left(P_{l o c}^{*} f(x)+p_{l o c}^{*} f(x)\right)
$$

where LP is a nonnegative kernel supported on $\tilde{N}$ and satisfying

$$
\sup _{x} \int_{\mathbb{R}^{d}} L P(x, y) d y<\infty \quad \text { and } \quad \sup _{y} \int_{\mathbb{R}^{d}} L P(x, y) d x<\infty .
$$

Consequently the integral operator associated to LP is of strong type $(p, p), 1 \leq p<\infty$, with respect to either Lebesgue or Gauss measure.

In order to prove the result above we need the following technical lemma, see [10] Lemma 3.4 .

Lemma 4.2 For $(x, y) \in \tilde{N}$ we have the following estimate

$$
D(x, y)=\int_{0}^{\infty}\left|O_{s}(x, y)-\chi_{(1, \infty)}(s) \frac{e^{-|y|^{2}}}{\pi^{d / 2}}-W_{s}(x, y)\right| \frac{d s}{s} \leq C\left(\frac{1+|x|^{\frac{1}{2}}}{|x-y|^{d-\frac{1}{2}}}+\log \frac{1}{|x-y|}\right) .
$$

In particular the above kernel when truncated by $\chi_{\tilde{N}}(x, y)$ satisfies conditions (4.19).

Proof of Lemma 4.1 By using Lemma 2.1 we have

$$
\begin{aligned}
\left\|U(\mathcal{P})_{l o c} f(x)-U(\Pi)_{l o c} f(x)\right\|_{E}=\left\|U\left(\mathcal{P}_{l o c}\right) f(x)-U\left(\Pi_{l o c}\right) f(x)\right\|_{E} \\
=\left\|\left\{P_{l o c, t_{i+1}} f(x)-P_{l o c, s} f(x)-\left(p_{l o c, t_{i+1}} f(x)-p_{l o c, s} f(x)\right)\right\}_{s \in J_{i}, i \in \mathbb{N}}\right\|_{E} \\
\leq\left\|\left\{P_{l o c, t_{i+1}} f(x)-P_{l o c, s} f(x)-\left(p_{l o c, t_{i+1}} f(x)-p_{l o c, s} f(x)\right)\right\}_{s \in J_{i}, i \in \mathbb{N}, t_{i} \leq 1}\right\|_{E} \\
+\left\|\left\{P_{l o c, t_{i+1}} f(x)-P_{l o c, s} f(x)-\left(p_{l o c, t_{i+1}} f(x)-p_{l o c, s} f(x)\right)\right\}_{s \in J_{i}, i \in \mathbb{N}, t_{i+1} \geq 1}\right\|_{E} \\
\quad+\left\|\left\{P_{l o c, t_{i+1}} f(x)-P_{l o c, s} f(x)-\left(p_{l o c, t_{i+1}} f(x)-p_{l o c, s} f(x)\right)\right\}_{s \in J_{i_{0}}, i_{0}=\min \left\{i: t_{i}>1\right\}}\right\|_{E} \\
\leq \quad\left\|\left\{P_{l o c, t_{i+1}} f(x)-P_{l o c, s} f(x)-\left(p_{l o c, t_{i+1}} f(x)-p_{l o c, s} f(x)\right)\right\}_{s \in J_{i}, i \in \mathbb{N}, t_{i} \leq 1}\right\|_{E} \\
\quad+\left\|\left\{P_{l o c, t_{i+1}} f(x)-P_{l o c, s} f(x)-\left(p_{l o c, t_{i+1}} f(x)-p_{l o c, s} f(x)\right)\right\}_{s \in J_{i}, i \in \mathbb{N}, t_{i+1} \geq 1}\right\|_{E} \\
\quad+2\left(P_{l o c}^{*} f(x)+p_{l o c}^{*} f(x)\right) \\
=\Lambda_{1}+\Lambda_{2}+2\left(P_{l o c}^{*} f(x)+p_{l o c}^{*} f(x)\right) .
\end{aligned}
$$

We observe that

$$
\int_{0}^{\infty}\left(1-\frac{u^{2}}{2 r}\right) e^{-u^{2} / 4 r} r^{-3 / 2} d r=0
$$


Now we shall analyze $\Lambda_{1}$. By using (1.2), (4.18) and (4.20) we have

$$
\begin{aligned}
& \sqrt{2 \pi} \Lambda_{1} \\
& =\left\|\left\{\int_{\mathbb{R}^{d}}\left(\int_{0}^{\infty}\left(t_{i+1} e^{-\frac{t_{i+1}^{2}}{4 r}}-s e^{-\frac{s^{2}}{4 r}}\right)\left(O_{r}(x, y)-W_{r}(x, y)\right) r^{-3 / 2} d r\right) \varphi(x, y) f(y) d y\right\}_{s \in J_{i}, i \in \mathbb{N}, t_{i} \leq 1}\right\|_{E}\left\|\left\{\int_{\mathbb{R}^{d}}\left(\int_{0}^{\infty}\left(\int_{t_{i+1}}^{s} e^{-\frac{u^{2}}{4 r}}\left(1-\frac{u^{2}}{2 r}\right) d u\right)\left(O_{r}(x, y)-W_{r}(x, y)\right) r^{-3 / 2} d r\right) \varphi(x, y) f(y) d y\right\}_{s \in J_{i}, i \in \mathbb{N}, t_{i} \leq 1}\right\|_{E}\|\| \\
& =\left\|\left\{\int_{\mathbb{R}^{d}}\left(\int_{t_{i+1}}^{s}\left(\int_{0}^{\infty} e^{-\frac{u^{2}}{4 r}}\left(1-\frac{u^{2}}{2 r}\right) r^{-\frac{3}{2}}\left(O_{r}(x, y)-\frac{e^{-|y|^{2}}}{\pi^{\frac{d}{2}}}-W_{r}(x, y)\right) d r\right) d u\right) \varphi(x, y) f(y) d y\right\}_{s \in J_{i}, i \in \mathbb{N}, t_{i} \leq 1}\right\|_{E} \\
& +\left\|\left\{\int_{\mathbb{R}^{d}}\left(\int_{0}^{1}\left(\int_{t_{i+1}}^{s} e^{-\frac{u^{2}}{4 r}}\left(1-\frac{u^{2}}{2 r}\right) d u\right) r^{-3 / 2} d r\right) \frac{e^{-|y|^{2}}}{\pi^{\frac{d}{2}}} \varphi(x, y) f(y) d y\right\}_{s \in J_{i}, i \in \mathbb{N}, t_{i} \leq 1}\right\|_{E} \int_{\mathbb{R}^{d}}\left(\int _ { 0 } ^ { \infty } ( \int _ { t _ { i + 1 } } ^ { s } e ^ { - \frac { u ^ { 2 } } { 4 r } } ( 1 - \frac { u ^ { 2 } } { 2 r } ) d u ) \left(O_{r}(x, y)-\chi_{(1, \infty)}(r) \frac{e^{-|y|^{2}}}{\pi^{\frac{d}{2}}}\right.\right. \\
& \left.\left.\left.\quad-W_{r}(x, y)\right) r^{-3 / 2} d r\right) \varphi(x, y) f(y) d y\right\}_{s \in J_{i}, i \in \mathbb{N}, t_{i} \leq 1} \|_{E} \\
& =\Lambda_{11}+\Lambda_{12} .
\end{aligned}
$$

To estimate $\Lambda_{11}$ we observe that, for $r$ in the range $1 \leq r<\infty$, we have

$$
\left\|\left\{\int_{t_{i+1}}^{s} e^{-\frac{u^{2}}{4 r}}\left(1-\frac{u^{2}}{2 r}\right) d u\right\}_{s \in J_{i}, i \in \mathbb{N}, t_{i} \leq 1}\right\|_{E} \leq C .
$$

In fact

$$
\begin{aligned}
& \left\|\left\{\int_{t_{i+1}}^{s} e^{-\frac{u^{2}}{4 r}}\left(1-\frac{u^{2}}{2 r}\right) d u\right\}_{s \in J_{i}, i \in \mathbb{N}, t_{i} \leq 1}\right\|_{E} \leq\left\|\left\{\int_{t_{i+1}}^{s} e^{-\frac{u^{2}}{4 r}} d u\right\}_{s \in J_{i}, i \in \mathbb{N}, t_{i} \leq 1}\right\|_{E} \\
& \leq\left(\sum_{i \in \mathbb{N}, t_{i} \leq 1}\left(\int_{t_{i+1}}^{t_{i}} e^{-\frac{u^{2}}{4 r}} d u\right)^{2}\right)^{1 / 2} \leq \sum_{i \in \mathbb{N}, t_{i} \leq 1} \int_{t_{i+1}}^{t_{i}} e^{-\frac{u^{2}}{4 r}} d u \\
& \leq \int_{0}^{1} e^{-\frac{u^{2}}{4 r}} d u=C \sqrt{r} \int_{0}^{1 / \sqrt{r}} e^{-t^{2}} d t \leq C,
\end{aligned}
$$

as claimed.

Therefore by using again (4.20) and (4.21) we have

$$
\begin{aligned}
\Lambda_{11} & =\left\|\left\{\int_{\mathbb{R}^{d}}\left(\int_{1}^{\infty}\left(\int_{t_{i+1}}^{s} e^{-\frac{u^{2}}{4 r}}\left(1-\frac{u^{2}}{2 r}\right) d u\right) r^{-3 / 2} d r\right) \frac{e^{-|y|^{2}}}{\pi^{\frac{d}{2}}} \varphi(x, y) f(y) d y\right\}_{s \in J_{i}, i \in \mathbb{N}, t_{i} \leq 1}\right\|_{E} \\
& \leq C \int_{\mathbb{R}^{d}}\left(\int_{1}^{\infty}\left\|\left\{\int_{t_{i+1}}^{s} e^{-\frac{u^{2}}{4 r}}\left(1-\frac{u^{2}}{2 r}\right) d u\right\}_{s \in J_{i}, i \in \mathbb{N}, t_{i} \leq 1}\right\|_{E} r^{-3 / 2} d r\right) e^{-|y|^{2}} \varphi(x, y)|f(y)| d y
\end{aligned}
$$




$$
\begin{aligned}
& \leq C \int_{\mathbb{R}^{d}}\left(\int_{1}^{\infty} r^{-3 / 2} d r\right) e^{-|y|^{2}} \varphi(x, y)|f(y)| d y \\
& \leq C \int_{\mathbb{R}^{d}} e^{-|y|^{2}} \varphi(x, y)|f(y)| d y .
\end{aligned}
$$

This proves the result for $\Lambda_{11}$.

In order to prove the result for $\Lambda_{12}$ we observe that, for any $r$ in the range $0<r<\infty$, we have

$$
\left\|\left\{\int_{t_{i+1}}^{s} e^{-\frac{u^{2}}{4 r}}\left(1-\frac{u^{2}}{2 r}\right) d u\right\}_{s \in J_{i}, i \in \mathbb{N}}\right\|_{E} \leq C r^{1 / 2} .
$$

In fact,

$$
\begin{gathered}
\left\|\left\{\int_{t_{i+1}}^{s} e^{-\frac{u^{2}}{4 r}}\left(1-\frac{u^{2}}{2 r}\right) d u\right\}_{s \in J_{i}, i \in \mathbb{N}}\right\|_{E} \leq\left(\sum_{i \in \mathbb{N}}\left(\int_{t_{i+1}}^{t_{i}} e^{-\frac{u^{2}}{4 r}}\left|1-\frac{u^{2}}{2 r}\right| d u\right)^{2}\right)^{1 / 2} \\
\leq \sum_{i \in \mathbb{N}} \int_{t_{i+1}}^{t_{i}} e^{-\frac{u^{2}}{4 r}}\left|1-\frac{u^{2}}{2 r}\right| d u \leq \int_{0}^{\infty} e^{-\frac{u^{2}}{4 r}}\left|1-\frac{u^{2}}{2 r}\right| d u \leq C r^{1 / 2} .
\end{gathered}
$$

Now by using (4.22) and Lemma 4.2 we have

$$
\Lambda_{12} \leq \int_{\mathbb{R}^{d}} C\left(\frac{1+|x|^{\frac{1}{2}}}{|x-y|^{d-\frac{1}{2}}}+\log \frac{1}{|x-y|}\right) \chi_{\tilde{N}}(x, y)|f(y)| d y .
$$

This gives the result for $\Lambda_{12}$.

In order to analyze $\Lambda_{2}$, we proceed exactly as for the case $\Lambda_{1}$ and we get $\Lambda_{2}=\Lambda_{21}+\Lambda_{22}$. Even more $\Lambda_{22}$ is estimated exactly as $\Lambda_{12}$. Therefore we only need to estimate $\Lambda_{21}$. By using that for any $\delta>0$, there exists $C$ such that $t e^{-t} \leq C t^{-\delta}$ and choosing $\delta>1 / 2$, we have

$$
\begin{aligned}
\Lambda_{21} & \leq C\left\|\left\{\int_{\mathbb{R}^{d}}\left(\int_{0}^{1}\left(\int_{t_{i+1}}^{s} e^{-\frac{u^{2}}{4 r}}\left|1-\frac{u^{2}}{2 r}\right| d u\right) r^{-3 / 2} d r\right) e^{-|y|^{2}} \chi_{\tilde{N}}(x, y)|f(y)| d y\right\}_{s \in J_{i}, i \in \mathbb{N}, t_{i+1} \geq 1}\right\|_{E} \\
& \leq C\left\|\left\{\int_{\mathbb{R}^{d}}\left(\int_{0}^{1}\left(\int_{t_{i+1}}^{s}\left(\frac{r}{u^{2}}\right)^{\delta} d u\right) r^{-3 / 2} d r\right) e^{-|y|^{2}} \chi_{\tilde{N}}(x, y)|f(y)| d y\right\}_{s \in J_{i}, i \in \mathbb{N}, t_{i+1} \geq 1}\right\|_{E} \\
& \leq C \int_{\mathbb{R}^{d}}\left(\int_{0}^{1}\left\|\left\{\left(\int_{t_{i+1}}^{s} u^{-2 \delta} d u\right)\right\}_{s \in J_{i}, i \in \mathbb{N}, t_{i+1} \geq 1}\right\|_{E} r^{-3 / 2+\delta} d r\right) e^{-|y|^{2}} \chi_{\tilde{N}}(x, y)|f(y)| d y \\
& \leq C \int_{\mathbb{R}^{d}}\left(\int_{0}^{1}\left(\sum_{i \in \mathbb{N}, t_{i+1} \geq 1} \int_{t_{i+1}}^{t_{i}} u^{-2 \delta} d u\right) r^{-3 / 2+\delta} d r\right) e^{-|y|^{2}} \chi_{\tilde{N}}(x, y)|f(y)| d y \\
& \leq C \int_{\mathbb{R}^{d}}\left(\int_{0}^{1}\left(\int_{1}^{\infty} u^{-2 \delta} d u\right) r^{-3 / 2+\delta} d r\right) e^{-|y|^{2}} \chi_{\tilde{N}}(x, y)|f(y)| d y \\
& \leq C \int_{\mathbb{R}^{d}} e^{-|y|^{2}} \chi_{\tilde{N}}(x, y)|f(y)| d y .
\end{aligned}
$$


This gives the result for $\Lambda_{2}$, ending the proof for the oscillation. As for the variation we remark that, as is easy to check, all the $E$-norm estimates remain true for the $F_{\rho}$-norm.

Proof of Theorem 1.2 By using formula (2.8) it is enough to prove that

$$
\|U(\mathcal{P}) f\|_{L_{E}^{p}\left(\mathbb{R}^{d}, d \gamma\right)} \leq C\|f\|_{L^{p}\left(\mathbb{R}^{d}, d \gamma\right)}
$$

and

$$
\gamma\left(\left\{x:\|U(\mathcal{P}) f(x)\|_{E}>\lambda\right\}\right) \leq \frac{C}{\lambda} \int_{\mathbb{R}^{d}}|f(x)| d \gamma(x) .
$$

Moreover, as $U(\mathcal{P}) f=U(\mathcal{P})_{\text {glob }} f+U(\mathcal{P})_{\text {loc }} f$ it suffices to prove the above inequalities for $U(\mathcal{P})_{g l o b} f$ and $U(\mathcal{P})_{l o c} f$.

By using Lemma 2.1 and (1.2), we have

$$
\begin{aligned}
\left\|U(\mathcal{P})_{g l o b} f(x)\right\|_{E} & =\left\|\left\{P_{t_{i+1}, g l o b} f(x)-P_{s, g l o b} f(x)\right\}_{s \in J_{i}, i \in \mathbb{N}}\right\|_{E} \\
& =\left\|\left\{\int_{t_{i+1}}^{s} \frac{\partial}{\partial t} P_{t, g l o b}(f)(x) d t\right\}_{s \in J_{i}, i \in \mathbb{N}}\right\|_{E} \\
& =\frac{1}{\sqrt{2 \pi}}\left\|\left\{\int_{t_{i+1}}^{s}\left(\int_{0}^{\infty}\left(1-\frac{t^{2}}{2 r}\right) e^{-\frac{t^{2}}{4 r}} r^{-3 / 2} O_{r, g l o b}(f)(x) d r\right) d t\right\}_{s \in J_{i}, i \in \mathbb{N}}\right\|_{E} \\
& =\frac{1}{\sqrt{2 \pi}}\left\|\left\{\int_{t_{i+1}}^{s}\left(\int_{0}^{\infty} \int_{0}^{r}\left(1-\frac{t^{2}}{2 u}\right) e^{-\frac{t^{2}}{4 u}} u^{-3 / 2} d u \frac{d}{d r} O_{r, g l o b}(f)(x) d r\right) d t\right\}_{s \in J_{i}, i \in \mathbb{N}}\right\|_{E} \\
& \leq \frac{1}{\sqrt{2 \pi}} \int_{0}^{\infty}\left\|\left\{\int_{t_{i+1}}^{s}\left|\int_{0}^{r}\left(1-\frac{t^{2}}{2 u}\right) e^{-\frac{t^{2}}{4 u}} u^{-3 / 2} d u\right| d t\right\}_{s \in J_{i}, i \in \mathbb{N}}\right\|_{E}\left|\frac{d}{d r} O_{r, g l o b}(f)(x)\right| d r .
\end{aligned}
$$

Making the change of variables $v=\frac{t}{2 \sqrt{u}}$, we have

$$
\int_{t_{i+1}}^{s}\left|\int_{0}^{r}\left(1-\frac{t^{2}}{2 u}\right) e^{-\frac{t^{2}}{4 u}} u^{-3 / 2} d u\right| d t=4 \int_{t_{i+1}}^{s}\left|\int_{\frac{t}{2 \sqrt{r}}}^{\infty}\left(1-2 v^{2}\right) e^{-v^{2}} d v\right| \frac{d t}{t} .
$$

Moreover

$$
\begin{aligned}
& \left\|\left\{\int_{t_{i+1}}^{s}\left|\int_{\frac{t}{2 \sqrt{r}}}^{\infty}\left(1-2 v^{2}\right) e^{-v^{2}} d v\right| \frac{d t}{t}\right\}_{s \in J_{i}, i \in \mathbb{N}}\right\|_{E}=\left\|\left\{\int_{t_{i+1}}^{s}\left|\left[v e^{-v^{2}}\right]_{t / 2 \sqrt{r}}^{\infty}\right| \frac{d t}{t}\right\}_{s \in J_{i}, i \in \mathbb{N}}\right\|_{E} \\
& \quad=\left\|\left\{\int_{t_{i+1}}^{s} \frac{t}{2 \sqrt{r}} e^{-\frac{t^{2}}{4 r}} \frac{d t}{t}\right\}_{s \in J_{i}, i \in \mathbb{N}}\right\|_{E} \leq\left(\sum_{i}\left(\int_{t_{i+1}}^{t_{i}} \frac{1}{2 \sqrt{r}} e^{-\frac{t^{2}}{4 r}} d t\right)^{2}\right)^{1 / 2} \\
& \leq \sum_{i} \int_{t_{i+1}}^{t_{i}} \frac{1}{2 \sqrt{r}} e^{-\frac{t^{2}}{4 r}} d t \leq \int_{0}^{\infty} \frac{1}{2 \sqrt{r}} e^{-\frac{t^{2}}{4 r}} d t \\
& =\int_{0}^{\infty} e^{-z^{2}} d z .
\end{aligned}
$$


Hence we have

$$
\begin{aligned}
\left\|U(\mathcal{P})_{g l o b} f(x)\right\|_{E} & \leq \frac{1}{\sqrt{2 \pi}} \int_{0}^{\infty}\left\|\left\{\int_{t_{i+1}}^{s}\left|\int_{0}^{r}\left(1-\frac{t^{2}}{2 u}\right) e^{-\frac{t^{2}}{4 u}} u^{-3 / 2} d u\right| d t\right\}_{s \in J_{i}, i \in \mathbb{N}}\right\|_{E}\left|\frac{d}{d r} O_{r, g l o b} f(x)\right| d r \\
& \leq C \int_{0}^{\infty}\left|\frac{d}{d r} O_{r, g l o b} f(x)\right| d r
\end{aligned}
$$

Now we make the change of parameter $e^{-r}=z$ and then we get

$$
\int_{0}^{\infty}\left|\frac{d}{d r} O_{r, g l o b} f(x)\right| d r=\int_{0}^{1}\left|\frac{d}{d z} T_{z, g l o b} f(x)\right| d z,
$$

where $T_{z}$ is the operator whose kernel is given by

$$
\mathcal{M}_{z}(x, y)=\left(\pi\left(1-z^{2}\right)\right)^{-d / 2} \exp \left(-\frac{|z x-y|^{2}}{1-z^{2}}\right) .
$$

We observe that the derivative of this kernel with respect to $z$ is the multiplication of a positive function times a polynomial of degree $3 \mathrm{in} z$. Then as a function of $z$, it changes sign at most three times (for a similar argument see the proof of Lemma 2.1 in [6]). So there exists a constant $C$ such that

$$
\begin{aligned}
\int_{0}^{1}\left|\frac{d}{d z} T_{z, g l o b} f(x)\right| d z & =\int_{0}^{1}\left|\frac{d}{d z} \int_{\mathbb{R}^{d}} \mathcal{M}_{z}(x, y)(1-\varphi(x, y)) f(y) d y\right| d z \\
& =\int_{0}^{1}\left|\int_{\mathbb{R}^{d}} \frac{d}{d z} \mathcal{M}_{z}(x, y)(1-\varphi(x, y)) f(y) d y\right| d z \\
& \leq \int_{0}^{1} \int_{\mathbb{R}^{d}}\left|\frac{d}{d z} \mathcal{M}_{z}(x, y)\right|(1-\varphi(x, y))|f(y)| d y d z \\
& =\int_{\mathbb{R}^{d}} \int_{0}^{1}\left|\frac{d}{d z} \mathcal{M}_{z}(x, y)\right| d z(1-\varphi(x, y))|f(y)| d y \\
& \leq C \int_{\mathbb{R}^{d}} \sup _{r}\left|O_{r}(x, y)\right|\left(\chi_{N^{c}}(x, y)\right)|f(y)| d y .
\end{aligned}
$$

This operator is known to be of weak type $(1,1)$ see [20]. It is also of strong type $(p, p)$, in the range $1<p<\infty$. Although the latter result is not explicitly given in the literature it can be obtained following the steps of the proof of Theorem 4.2 in [16] and using the estimate for the global part of the kernel given in [13].

For the local part, by Lemma (4.1) we have

$$
\begin{aligned}
\left\|U(\mathcal{P})_{l o c} f(x)\right\|_{E} & \leq\left\|U(\mathcal{P})_{l o c} f(x)-U(\Pi)_{l o c} f(x)\right\|_{E}+\left\|U(\Pi)_{l o c} f(x)\right\|_{E} \\
& \leq \int_{\mathbb{R}^{d}} L P(x, y) f(y) d y+2 P_{l o c}^{*} f(x)+2 p_{l o c}^{*} f(x)+\left\|U(\Pi)_{l o c} f(x)\right\|_{E}
\end{aligned}
$$

The first term of this summation, according to Lemma 4.1 , is bounded in $L^{p}\left(\mathbb{R}^{d}, d \gamma\right)$ for every $p$ in the range $1 \leq p<\infty$. The second term is bounded by a multiple of $P^{*}$ which 
is known to satisfy the desired estimates. To handle the third term we first observe that $p_{l o c}^{*} f(x)$ is pointwise bounded by $p^{*} f(x)$ that has the corresponding boundedness properties with respect to the Lebesgue measure; therefore as $p_{l o c}^{*}$ is a local operator we may apply Proposition 2.2 to get the right estimate with respect to the Gaussian measure. As for the fourth term it is known, see [3], that

$$
\left\|\mathcal{O}^{\prime}(\Pi f)\right\|_{L^{p}\left(\mathbb{R}^{d}, d x\right)} \leq C\|f\|_{L^{p}\left(\mathbb{R}^{d}, d x\right)}
$$

and

$$
\left|\left\{x:\left|\mathcal{O}^{\prime}(\Pi f)(x)\right|>\lambda\right\}\right| \leq \frac{C}{\lambda} \int_{\mathbb{R}^{d}}|f(x)| d x .
$$

That is, by using (2.8),

$$
\|U(\Pi f)\|_{L_{E}^{p}\left(\mathbb{R}^{d}, d x\right)} \leq C\|f\|_{L^{p}\left(\mathbb{R}^{d}, d x\right)}
$$

and

$$
\left|\left\{x:\|U(\Pi) f(x)\|_{E}>\lambda\right\}\right| \leq \frac{C}{\lambda} \int_{\mathbb{R}^{d}}|f(x)| d x .
$$

In order to apply Proposition 2.1 to the operator $U(\Pi)$, we observe that its kernel is given by

$$
\mathcal{U}(x, y)=\left\{\left(p_{t_{i+1}}(x, y)-p_{s}(x, y)\right) \chi_{J_{i}(s)}\right\} .
$$

By using (4.18), and following the ideas developed in (4.25) we have

$$
\begin{aligned}
\mathcal{U}(x, y) & =\left\{\int_{t_{i+1}}^{s} \frac{\partial}{\partial t} p_{t}(x, y) d t\right\}_{s \in J_{i}, i \in \mathbb{N}}=\left\{\int_{t_{i+1}}^{s} \int_{0}^{\infty}\left(1-\frac{t^{2}}{2 r}\right) e^{-\frac{t^{2}}{4 r}} r^{-3 / 2} W_{r}(x, y) d r d t\right\}_{s \in J_{i}, i \in \mathbb{N}} \\
& =\left\{\int_{t_{i+1}}^{s} \int_{0}^{\infty}\left(\int_{0}^{r}\left(1-\frac{t^{2}}{2 u}\right) e^{-\frac{t^{2}}{4 u}} u^{-3 / 2} d u\right) \frac{d}{d r} W_{r}(x, y) d r d t\right\}_{s \in J_{i}, i \in \mathbb{N}} .
\end{aligned}
$$

Therefore,

$$
\begin{aligned}
\|\mathcal{U}(x, y)\|_{E} & =\left\|\left\{\int_{t_{i+1}}^{s} \int_{0}^{\infty}\left(\int_{0}^{r}\left(1-\frac{t^{2}}{2 u}\right) e^{-\frac{t^{2}}{4 u}} u^{-3 / 2} d u\right) \frac{d}{d r} W_{r}(x, y) d r d t\right\}_{s \in J_{i}, i \in \mathbb{N}}\right\|_{E} \\
& \leq \int_{0}^{\infty}\left\|\left\{\int_{t_{i+1}}^{s}\left|\int_{0}^{r}\left(1-\frac{t^{2}}{2 u}\right) e^{-\frac{t^{2}}{4 u}} u^{-3 / 2} d u\right| d t\right\}_{s \in J_{i}, i \in \mathbb{N}}\right\|_{E}\left|\frac{d}{d r} W_{r}(x, y)\right| d r \\
& \leq \int_{0}^{\infty}\left\|\left\{\int_{t_{i+1}}^{s}\left|\int_{0}^{r}\left(1-\frac{t^{2}}{2 u}\right) e^{-\frac{t^{2}}{4 u}} u^{-3 / 2} d u\right| d t\right\}_{s \in J_{i}, i \in \mathbb{N}}\right\|_{E}\left|\frac{d}{d r} W_{r}(x, y)\right| d r \\
& \leq C \int_{0}^{\infty}\left|\frac{d}{d r} W_{r}(x, y)\right| d r=C \int_{0}^{\infty}\left|\frac{d}{d r} \frac{1}{r d / 2} e^{-\frac{|x-y|^{2}}{r}}\right| d r \\
& \leq \frac{C}{|x-y|^{d}} .
\end{aligned}
$$

Hence we are in the hypothesis of Proposition 2.1 and consequently we have

$$
\left\|U\left(\Pi_{l o c} f\right)\right\|_{L_{E}^{p}\left(\mathbb{R}^{d}, d x\right)} \leq C\|f\|_{L^{p}\left(\mathbb{R}^{d}, d x\right)}
$$


and

$$
\left|\left\{x:\left\|U(\Pi)_{l o c} f(x)\right\|_{E}>\lambda\right\}\right| \leq \frac{C}{\lambda} \int_{\mathbb{R}^{d}}|f(x)| d x .
$$

Since we are dealing with a local operator Proposition 2.2 applies giving the desired estimates for the oscillation. As before, we observe that the estimates and results used in the above proof are also true for the variation operator as long as $2<\rho<\infty$.

This ends the proof of Theorem 1.2.

Note added in review: We thank the referee for bringing our attention to the article [12] where results related to those in Section 4 are proved and for pointing out some missprints.

\section{References}

[1] D. G. Austin, A sample function property for martingales, Ann. Math. Statist. 37 (1966), 1396-1397.

[2] J. Bourgain, Pointwise ergodic theorems for arithmetic sets, Inst. Hautes Etudes Sci. Publ. Math. 69 (1989), 5-45.

[3] J.T. Campbell, R.L. Jones, K. Reinhold and M. Wierdl, Oscillation and variation for the Hilbert transform, Duke Math. J. 105 (2000), 59- 83.

[4] J.T. Campbell, R.L. Jones, K. Reinhold and M. Wierdl, Oscillation and variation for singular integrals in higher dimensions, Trans. Amer. Math. Soc. 355(2003) 2115-2137.

[5] E. Fabes, C. Gutiérrez and R. Scotto, Weak-type estimates for the Riesz transforms associated with the Gaussian measure, Rev. Mat. Iberoamericana 10 (1994), 229-281.

[6] J. García-Cuerva, G. Mauceri, P. Sögren and J.L. Torrea, Spectral multipliers for the Ornstein-Uhlenbeck semigroup, J. Anal. Math. 78 (1999), 281-305.

[7] T. A. Gillespie and J.L. Torrea, Dimension free estimates for the oscillation of Riesz transforms, Israel J. Math. (to appear).

[8] R. Gundy, Some topics in probability and analysis, CBMS Regional Conf. Ser. in Math. 70, American Mathematical Society, Providence, RI 1989.

[9] C.E. Gutiérrez, On the Riesz transforms for Gaussian measures, J. Funct. Anal. 120 (1994), 107-134.

[10] E.Harboure, J.L. Torrea and B. Viviani, Vector valued extensions of operators related to the Ornstein-Uhlenbeck semigroup, J. Anal. Math., to appear.

[11] R. Jones, R. Kaufman, J. Rosenblatt and M. Wierdl, Oscillation in ergodic theory, Erg. Th. \& Dynam. Sys. 18 (1998), 889-936. 
[12] R. Jones and K. Reinhold Oscillation and variation inequalities for convolution powers, Erg. Th. \& Dynam. Sys. 21 (2001), 1809-1829.

[13] T. Menárguez, S. Pérez and F. Soria, The Mehler maximal funtion: a geometric proof of the weak type 1, J. London Math. Soc. 61 (2000), 846-856.

[14] P.A. Meyer, Transformations de Riesz pour le lois Gaussienes, Lecture Notes in Math. 1059 (1984), 179-193. Springer-Verlag. New York.

[15] B. Muckenhoupt, Poisson integrals for Hermite and Laguerre expansions, Trans. Amer. Math. Soc. 139 (1969), 231-242.

[16] S. Pérez, The local part and the strong type for operators related to the Gaussian measure, J. Geom. Anal. 11 (2001), 491-507.

[17] S. Pérez and F. Soria, Operators associated with the Ornstein-Uhlenbeck semigroup, J. London Math. Soc. 61 (2000), 857-871.

[18] G. Pisier, Riesz transforms: a simpler proof of P.A. Meyer's inequality, Springer Lecture Notes in Mathematics 1321 (1988), 485-501.

[19] J.L. Rubio de Francia, F.J. Ruiz and J.L. Torrea, Calderón-Zygmund kernels for operator-valued kernels, Adv. Math. 62 (1986), 7-48.

[20] P. Sjögren, On the maximal function for the Mehler kernel, Lecture Notes in Math. 992 (1983), 73-82.

[21] E.M. Stein, Topics in Harmonic Analysis Related to the Littlewood-Paley Theory, Annals of Math. Studies, vol. 63, Princeton U. Press, Princeton, NJ, 1970.

[22] E.M. Stein, Singular Integrals and Differentiability Properties of Functions, Princeton Univ. Press, Princeton, NJ, 1970.

[23] W.O. Urbina, On singular integrals with respect to the Gaussian measure, Ann. Scuola Norm. Sup. Pisa Cl. Sci. 17 (1990), 531-567.

Departamento de Matemática,

Facultad de Ingeniería Química,

Universidad Nacional del Litoral

(3000) Santa Fe y CONICET, Argentina.

e-mail: harbour@ceride.gov.ar

e-mail:rmacias@ceride.gov.ar

E.T.S. I. de Caminos, Canales y Puertos,

Universidad Politécnica de Madrid 
Ciudad Universitaria s/n

28040 Madrid, Spain.

e-mail: tmenar@dumbo.caminos.upm.es

Facultad de Ciencias,

Universidad Autónoma de Madrid

Ciudad Universitaria de Cantoblanco

28049 Madrid, Spain.

e-mail: joseluis.torrea@uam.es 